\title{
YTHDF1 promotes the proliferation, migration, and invasion of prostate cancer cells by regulating TRIM44
}

\author{
Weijian $\mathrm{Li}^{1}$ - Gaohuang Chen ${ }^{1} \cdot$ Zhenyu Feng $^{1}$ - Baoyi Zhu ${ }^{1}$ - Lilin Zhou ${ }^{1} \cdot$ Yuying Zhang ${ }^{1}$ Junyan Mai ${ }^{1}$. \\ Chonghe Jiang ${ }^{1}$. Jianwen Zeng ${ }^{1,2}$
}

Received: 28 June 2021 / Accepted: 30 September 2021 / Published online: 22 October 2021

(c) The Author(s) 2021

\begin{abstract}
Background Prostate cancer (PCa) is one of the most common malignancies in men. YTHDF1 may play an important role in promoting PCa progression, but there is no reports to date on YTHDF1 function in PCa.

Objective This study explored whether YTHDF1 could regulate TRIM44 in PCa cells.

Methods By querying the TCGA database, we evaluated YTHDF1 expression in PCa, the OS and DFS of YTHDF1, and the correlation between YTHDF1 and TRIM44 in PCa. We constructed vectors to interfere with YTHDF1 expression and overexpress TRIM44 to examine the role of YTHDF1 and TRIM44 in PCa cells. Differentially expressed mRNAs were identified by mRNA sequencing. The levels of YTHDF1, TRIM44, LGR4, SGTA, DDX20, and FZD8 were measured by qRT-PCR and WB was used to determine YTHDF1 and TRIM44 expression. A CCK-8 assay was used to assess cell proliferation. A Transwell chamber assay was used measure cell migration and invasion ability.

Results YTHDF1 was highly expressed in both Pca tissues and cells. PCa patient prognosis with high YTHDF1 expression was relatively poor. Cell function experiments showed that inhibiting YTHDF1 expression decreased cell proliferation, migration, and invasion. RNA sequencing analysis revealed that YTHDF1 may promote PCa cell proliferation, migration, and invasion by modulating TRIM44 expression. Cell function experiments further verified that YTHDF1 promoted PCa cell proliferation, migration, and invasion by regulating TRIM44.
\end{abstract}

Conclusions YTHDF1 enhances PCa cell proliferation, migration, and invasion by regulating TRIM44.

Keywords YTHDF1 $\cdot$ TRIM44 $\cdot$ Prostate cancer cells $\cdot$ Migration $\cdot$ Invasion

\section{Introduction}

Prostate cancer (PCa) is the most common cancer of solid organs in men and the second most common cause of cancerrelated deaths in men (Nguyen-Nielsen and Borre 2016). PCa can present with a variety of clinical manifestations ranging from inert microscopic disease to highly aggressive

Weijian Li and Gaohuang Chen contributed equally to this work.

Jianwen Zeng

zengjwen@gzhmu.edu.cn

1 Department of Urology, The Sixth Affiliated Hospital of Guangzhou Medical University (Qingyuan People's Hospital), Qingyuan 511518, Guangdong, China

2 Department of Urology, The Sixth Affiliated Hospital of Guangzhou Medical University (Qingyuan People's Hospital), B24 Yinquan Road, Qingcheng, Qingyuan 511500, Guangdong, China tumors, including a tendency to metastasize (Lancia et al. 2019). A significant problem for PCa patients is the detection of recurrent disease and the treatment of metastatic cancer (Haberkorn et al. 2016). Most PCa patients receive prostatectomy, radiotherapy, chemotherapy, or hormone therapy, however, the 5-year recurrence rate is approximately $30 \%$ (Jemal et al. 2007; Tian et al. 2018). Despite new progress, PCa remains a significant medical problem for men. The high morbidity and mortality of PCa require more work to uncover the underlying mechanisms. In addition, advances in the treatment of PCa require new biomarkers with high accuracy for diagnosis and risk stratification to improve response and outcome.

N6-Methyladenosine (m6A) is the most abundant internal modification of RNA in eukaryotic cells and it regulates mRNA splicing, translation, and stability (Sun et al. 2019). Recent studies indicate that m6A contributes to tumorigenesis through multiple mechanisms (Zhang et al. 2021). Three types 
of enzymes regulate m6A modification, which include "Writers" (mA methyltransferases including METTL3), "Erasers" (mA demethylases including FTO), and "readers" (mA binding proteins including YTH domain proteins YTHDF1, F2, F3, and heterogeneous nuclear ribonucleoproteins, hnRNPs) (Liang et al. 2020; Meyer and Jaffrey 2017). The "reader" proteins contribute to RNA metabolism through the m6A signal (Xia et al. 2021). YTHDF1 promotes translation efficiency of m6A-modified mRNAs and is associated with a variety of cancers (Wang et al. 2015). YTHDF1 promotes ovarian cancer progression by regulating EIF3C translation (Liu et al. 2020) and plays an important role in other cancers including lung, gastrointestinal, liver, and oral cancers (Nishizawa et al. 2018; Shi et al. 2019; Zhao et al. 2018, 2020). m6A plays an important role in PCa and YTHDF2 is involved in regulating PCa progression ( $\mathrm{Li}$ et al. 2018). TCGA data indicate that YTHDF1 expression in $\mathrm{PCa}$ is higher compared with normal tissues. The prognosis and survival rate of PCa patients with high expression is lower, suggesting that YTHDF1 may play a role in promoting PCa progression. However, there are no studies to date describing a function of YTHDF1 in PCa. Therefore, it is necessary to validate the expression and function of YTHDF1 in PCa.

Tripartite motif containing 44 (TRIM44), an important member of the TRIM family, contributes to a variety of pathological states and is closely associated with malignant tumor etiology and progression (Chen et al. 2021a; Liu et al. 2019; Sato et al. 2021). In addition, TRIM44 gene knockout inhibits PCa cell proliferation and invasion (Tan et al. 2017). These findings suggest that TRIM44 may serve as a prognostic marker and a new therapeutic target for PCa. TCGA data indicate that YTHDF1 and TRIM44 are significantly correlated in $\mathrm{PCa}$ and they are both up-regulated. YTHDF1 may promote PCa cell proliferation, migration, and invasion by modulating TRIM44 expression. However, whether YTHDF1 plays a role in PCa cells by regulating TRIM44 remains unclear.

In the present study, we analyzed YTHDF1 expression in PCa and discuss the role of YTHDF1 and TRIM44 in PCa progression. We show that YTHDF1 expression is significantly up-regulated in PCa tissues and cells. Interfering with YTHDF1 expression inhibits cell proliferation, migration, and invasion. Furthermore, YTHDF1 promotes PCa cells proliferation, migration, and invasion by regulating TRIM44. Overall, our study provides a prognostic biomarker and a new therapeutic target for PCa.

\section{Materials and methods}

\section{Cell culture and transfection}

WPMY-1 human normal prostate stromal immutable cells and human PCa cells, PC3, LNCAP, 22RV1, and
PC-3 M-IE8 were purchased from the ATCC. The culture conditions for WPMY-1 were DMEM, high glucose, and 5\% fetal bovine serum (FBS). PC3 was cultured using F-12 K containing 10\% FBS. The culture conditions for LNCAP, 22RV1, and PC-3 M-IE8 consisted of RPMI 1640 containing $10 \%$ FBS. To interfere with the expression of YTHDF1, si-YTHDF1 was transfected into the human PCa cell lines, PC3 and LNCap, for $48 \mathrm{~h}$, and then divided into PC3 + si-NC, PC3 + si-YTHDF1, LNCap + si-NC, and LNCap + si-YTHDF1 groups. To investigate whether YTHDF1 plays a role in PCa by regulating TRIM44, we conducted recovery experiments in $\mathrm{PC} 3$ cells, which were divided into $\mathrm{PC} 3$ + si-NC, PC3 + si-YTHDF1, and PC3 + si-YTHDF1 + TRIM44 groups. si-YTHDF1 (GCUGGCAAAUAUGAA GGUATT) and si-NC (UUCUCCGAACGUGUCACGUTT) constructs were designed and synthesized by GenePharma (Shanghai, China). To overexpress TRIM44, the TRIM44 sequence was cloned into the LV003 vector (Cookgen, Guangzhou, China). Cell transfection was performed using Lipofectamine 2000 (Invitrogen) according to the manufacturer's instructions.

\section{Quantitative Real-time PCR (qRT-PCR)}

YTHDF1, TRIM44, LGR4, SGTA, DDX20, and FZD8 expression in cells were measured by qRT-PCR. Briefly, total RNA was extracted by the Trizol method, cDNA was reverse-transcribed (Takara, Japan) into cDNA, and the cDNA products were used for qPCR using the SYBR Green Premix kit (Takara, Japan). GAPDH was used as the reference gene and relative gene expression was calculated by the $2^{-\Delta \Delta \mathrm{C}_{\mathrm{T}}}$ method. The primers used in this study are shown in Table 1.

\section{Western blot (WB) analysis}

RIPA lysis buffer was used to prepare protein extracts from cells and tissues and the protein was quantitated using the BCA protein assay kit. The samples were mixed with SDSPAGE loading buffer and heated in a boiling water bath at $100{ }^{\circ} \mathrm{C}$ for $5 \mathrm{~min}$. The proteins were transferred to PVDF membranes, blocked for $2 \mathrm{~h}$ with $5 \%$ skim milk at room temperature, and incubated with YTHDF1 (86,463, CST), TRIM44 (AB236422, ABCAM), and GAPDH (60004-1-LG, Proteintech) primary antibodies for $90 \mathrm{~min}$ at room temperature. The membranes were washed three times with TBST and the secondary antibodies were added. The protein bands were developed by chemiluminescence and measured with an Odyssey Infrared Imaging System (Li-Cor Biosciences, Lincoln, NE, USA). GAPDH was used as the internal reference to quantitate relative protein expression levels. 
Table 1 The primers used in this study

\begin{tabular}{ll}
\hline Primer ID & $5^{\prime}-3^{\prime}$ \\
\hline YTHDF1-F & CAGCACCGATCCCGACATAG \\
YTHDF1-R & CTGGCTTCCTGAAGACGATGA \\
TRIM44-F & GCCAGGAAGATAGGCAGCTCAT \\
TRIM44-R & CTTCAGTCCACCTGAGTCTTTGC \\
LGR4-F & GGAGCATTTGATGGTAATCCACTC \\
LGR4-R & CCATGCTTGCACCACGAATGAC \\
SGTA-F & CGGTAGAAGACAGTGACCTTGC \\
SGTA-R & TCTGCTGAGTCCTCCTCGGAAG \\
DDX20-F & AATCAGCGTCTTGATGCTATGGC \\
DDX20-R & ACAACCAGATTCACCTTCTCAGC \\
FZD8-F & GCTCTACAACCGCGTCAAGACA \\
FZD8-R & AAGGTGGACACGAAGCAGAGCA \\
GAPDH-F & GAGTCAACGGATTTGGTCGT \\
GAPDH-R & GACAAGCTTCCCGTTCTCAG \\
\hline
\end{tabular}

\section{Cell counting kit 8 (CCK-8) assay}

The cells were digested with trypsin. The cell suspension was seeded into 96 -well plates at a density of $5 \times 10^{3}$ cells/ well. Three replicates were established for each group for treatment according to the experimental groups. The cells were cultured for $24 \mathrm{~h}$. After incubation for a designated period of time, the medium was discarded and replaced with $10 \mu \mathrm{L} \mathrm{CCK}-8$ working solution and incubated at $37{ }^{\circ} \mathrm{C}$ and $5 \% \mathrm{CO}_{2}$ for another $4 \mathrm{~h}$. The absorbance was measured using an ELX800 microplate reader (BioTek, Winooski, Vermont, USA) at $450 \mathrm{~nm}$. The proliferation ability of cells at 24,48 , and $72 \mathrm{~h}$ was determined.

\section{Cell migration assay}

PC3 and LNCaP cell migration were measured by a cell migration assay. Cells $\left(1 \times 10^{6} / \mathrm{mL}\right)$ were resuspended in serum-free medium and $100 \mu \mathrm{L}$ of cell suspension was added to the upper compartment of a Transwell chamber (\#33318035, Corning). Complete medium containing 10\% FBS was added to the lower chamber and cultured for $48 \mathrm{~h}$. The culture medium was discarded and the chamber was washed twice with PBS. After wiping the upper compartment with a wet cotton swab, they were fixed with $4 \%$ paraformaldehyde for $10 \mathrm{~min}$, stained with $0.5 \%$ crystal violet for $5 \mathrm{~min}$, and rinsed with water. The cells on the upper and outer surfaces were observed and imaged under a microscope (Olympus, Japan).

\section{Cell invasion assay}

The inner side of the upper chamber of a Transwell chamber was precoated with Matrigel Basement Membrane Matrix
(BD Biocoat). The Matrigel was dissolved overnight at $4{ }^{\circ} \mathrm{C}$ and then diluted 1:3 with precooled base medium (Matrigel: Medium). Then, $40 \mu \mathrm{L}$ of Matrigel was added to the precooled Transwell chamber and incubated at $37{ }^{\circ} \mathrm{C}$ for $2 \mathrm{~h}$ to solidify. The cells were counted with basal medium, adjusted to $1 \times 10^{6} / \mathrm{mL}$, and $100 \mu \mathrm{L}$ and $600 \mu \mathrm{L}$ complete medium was added to the upper and lower compartments of the Transwell chamber. After incubating at $37^{\circ} \mathrm{C}$ for $48 \mathrm{~h}$, the cells in the upper compartment were wiped with a wet cotton swab, fixed with $4 \%$ paraformaldehyde for $10 \mathrm{~min}$, and stained with $0.5 \%$ crystal violet for $5 \mathrm{~min}$. A microscope (Olympus, Japan) was used to observe whether the cells entering the bottom cavity through the pore, and images were captured.

\section{mRNA sequencing and analysis}

To further understand the YTHDF1 regulatory mechanism in $\mathrm{PCa}$, transcriptome sequencing analysis was performed on PC3_siRNA_NC and PC3_siRNA_YTHDF1 groups. The RNA sequencing was done by Novogene Bioinformatics Technology Co., Ltd. (Beijing, China). We used an Illumina $\times 10$ sequencing instrument and the average sequencing depth was $100 \times$. The $\mathrm{R}$ software package was used for quantile normalization to identify differentially expressed mRNAs. The screening criteria were $\log \mathrm{FCl}>1$ and $\mathrm{P}<0.05$. Expression patterns of the mRNA were obtained by hierarchical clustering. Gene ontology (GO) and Kyoto Encyclopedia of Genes and Genome (KEGG) analysis were also performed.

\section{Bioinformatic analysis}

By querying the TCGA database (http://starbase.sysu.edu.cn/ panGeneDiffExp.php) and (http://gepia.cancer-pku.cn/detail. php? gene $=$ YTHDF1), we analyzed YTHDF1 expression in PCa. The overall survival (OS) and disease-free survival (DFS) of YTHDF1 and the correlation between YTHDF1 and TRIM44 in PCa were determined.

\section{Statistical analysis}

Graphpad 8.0 was used for statistical analysis and the experimental data were expressed as the mean $\pm \mathrm{SD}$, which were measured in triplicate. Differences between two groups were analyzed by the Student's t test. One-way ANOVA was used for comparisons between more than two groups. $\mathrm{P}<0.05$ was considered statistically significant. 


\section{Results}

\section{YTHDF1 is highly expressed in PCa cells}

To detect YTHDF1 expression in PCa, we compared 492 PCa tissues $(\mathrm{T})$ and 152 paracancerous tissues $(\mathrm{N})$ by querying the TCGA database and found that YTHDF1 was highly expressed in $\mathrm{PCa}(\mathrm{P}=0.003$, Fig. 1A). Figure 1B C shows the OS and DFS for YTHDF1 in PCa. The results indicate that PCa patients with high YTHDF1 expression have a relatively poor prognosis. To further verify YTHDF1 expression in PCa, qRT-PCR and WB were performed to measure the expression of YTHDF1 in PCa cells and WPMY-1 cells (Fig. 1D, E). The results indicate that YTHDF1 expression in PCa cells was higher compared with that in WPMY-1 cells. These results indicate that YTHDF1 was highly expressed in both PCa tissues and cells and increased expression results in a worse prognosis, which suggests that YTHDF1 may function as a proto-oncogene.

\section{Suppressing YTHDF1 expression inhibits cell proliferation, migration, and invasion}

To investigate the effects of YTHDF1 on PCa cells, we transfected si-YTHDF1 interference fragments into PC3 and LNCap cells. As shown in Fig. 2A, YTHDF1 expression was significantly inhibited indicating successful transfection. Cell function experiments revealed that cell proliferation in the si-YTHDF1 group was significantly decreased, whereas the number of migrating and invading cells was reduced compared with the si-NC group (Fig. 2B, D). These results indicate that interfering with the expression of YTHDF1 reduces $\mathrm{PCa}$ cells proliferation, migration, and invasion.

\section{mRNA sequencing analyzing the regulatory mechanism of YTHDF1 in PCa}

To further understand the YTHDF1 regulatory mechanism in $\mathrm{PCa}$, we performed mRNA sequencing analysis. The results showed that following YTHDF1 interference, a total of 2992 genes were significantly up-regulated and 2509 genes were significantly down-regulated in PC3 cells (Fig. 3A). GO analysis revealed that the differentially expressed genes were divided into three categories. Among the biological processes, the differentially expressed genes included

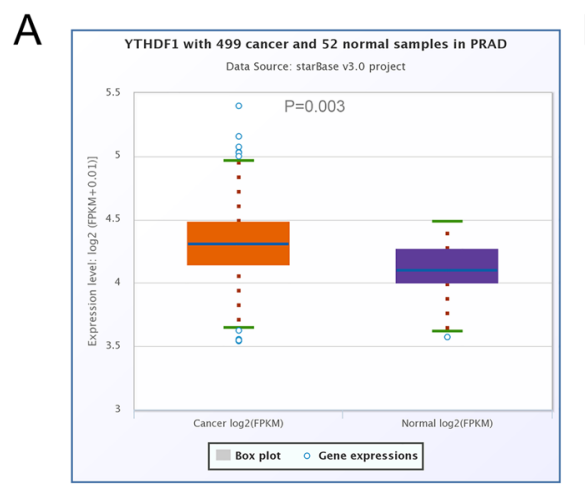

D

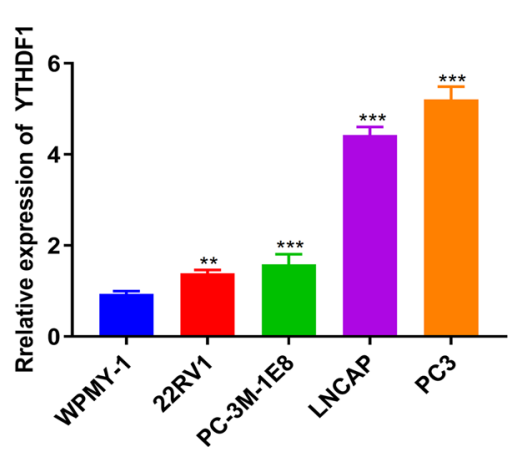

B

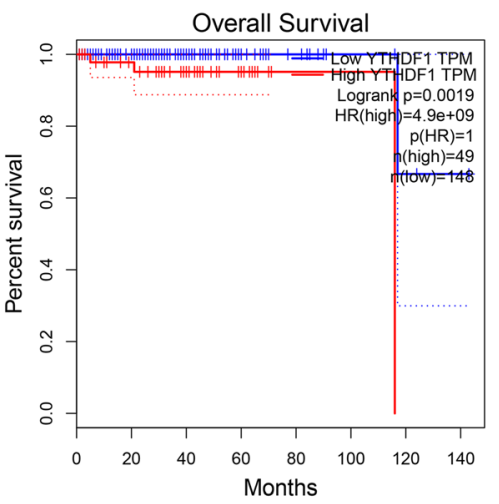

E

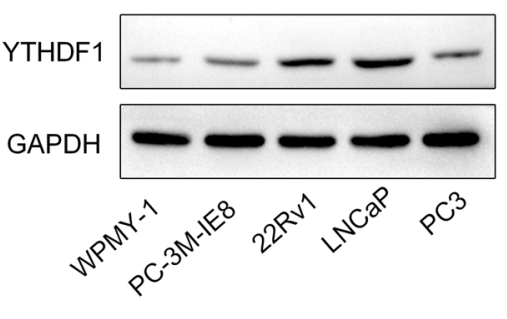

C
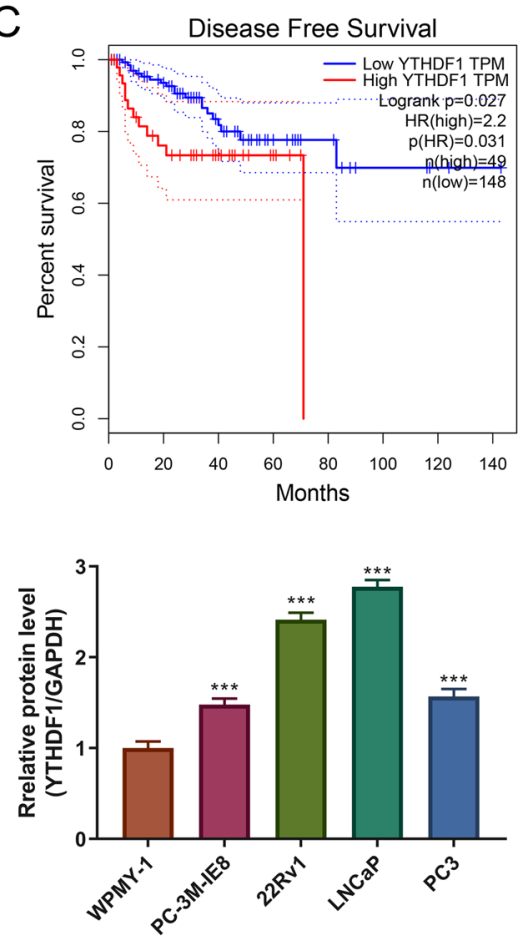

Fig. 1 High expression of YTHDF1 in prostate cancer cells. A YTHDF1 expression in prostate cancer tissues and paracancerous tissues. B The OS of YTHDF1 in PCa. C The DFS of YTHDF1 in PCa. D qRT-PCR measurement of YTHDF1 expression in WPMY-1 and PCa cells (PC3, LNCap, 22RV1, and PC-3 M-IE8). E Western blotting was performed to detect YTHDF1 protein expression in WPMY-1 and prostate cancer cells (PC3, LNCap, 22RV1, and PC-3 M-IE8). $* \mathrm{P}<0.05, * * \mathrm{P}<0.005, * * * \mathrm{P}<0.001$ 
A

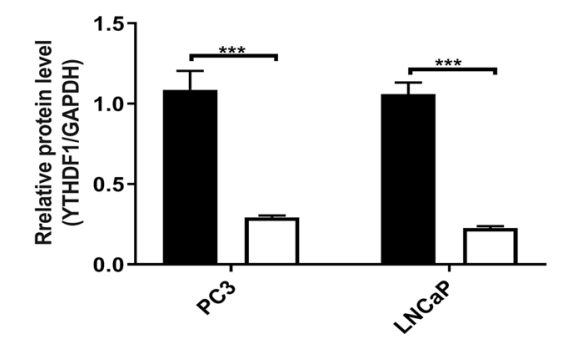

C

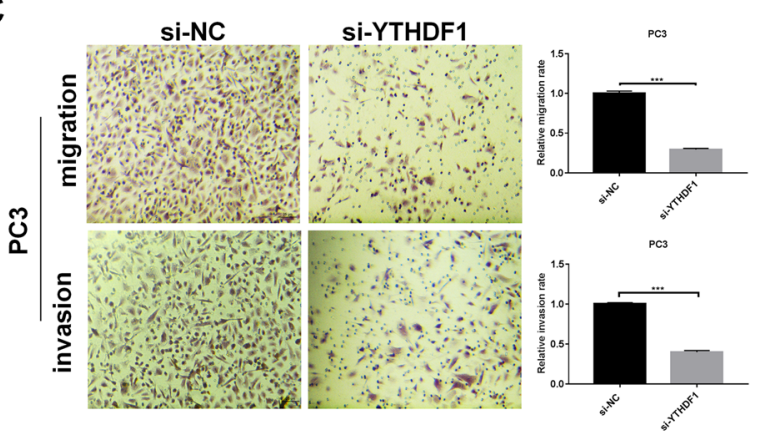

Fig. 2 Interfering with YTHDF1 inhibits prostate cancer cell proliferation, migration, and invasion. A qRT-PCR measurement of YTHDF1 transfection efficiency. B CCK-8 assay was performed to detect PC3 and LNCap cell proliferation at 24, 48, and 72 h. C PC3

cellular process, single-organism process, and metabolic regulation. For the cellular component classification, the differentially expressed genes were mainly divided into the cell, cell part, organelle part, and membrane. For the classification of molecular function, differentially expressed genes were mainly divided into the binding, catalytic activity, and molecular function regulator (Fig. 3B). Using KEGG, we analyzed the top 20 signaling pathways and identified PI3KAkt pathway, which plays a major role in regulating cell proliferation, growth, differentiation, and apoptosis (Fig. 3C). We selected five genes (TRIM44, LGR4, SGTA, DDX20, and FZD8) that have been reported previously in PCa and are considered significant differentially expressed genes. qRT-PCR and WB revealed TRIM44 mRNA and protein expression were significantly down-regulated in the si-YTHDF1 group (Fig. 3D, E). The TCGA database indicated that YTHDF1 and TRIM44 are significantly correlated in PCa, and they were both up-regulated (Fig. 3F). This suggests that YTHDF1 may promote PCa cells proliferation, migration, and invasion by modulating TRIM44 expression.

\section{YTHDF1 regulates TRIM44 to promote PCa cells proliferation, migration, and invasion}

To explore whether YTHDF1 functions by regulating TRIM44 in PCa cells, we performed recovery experiments in PC3 cells. As shown in Fig. 4A, after overexpression of
B
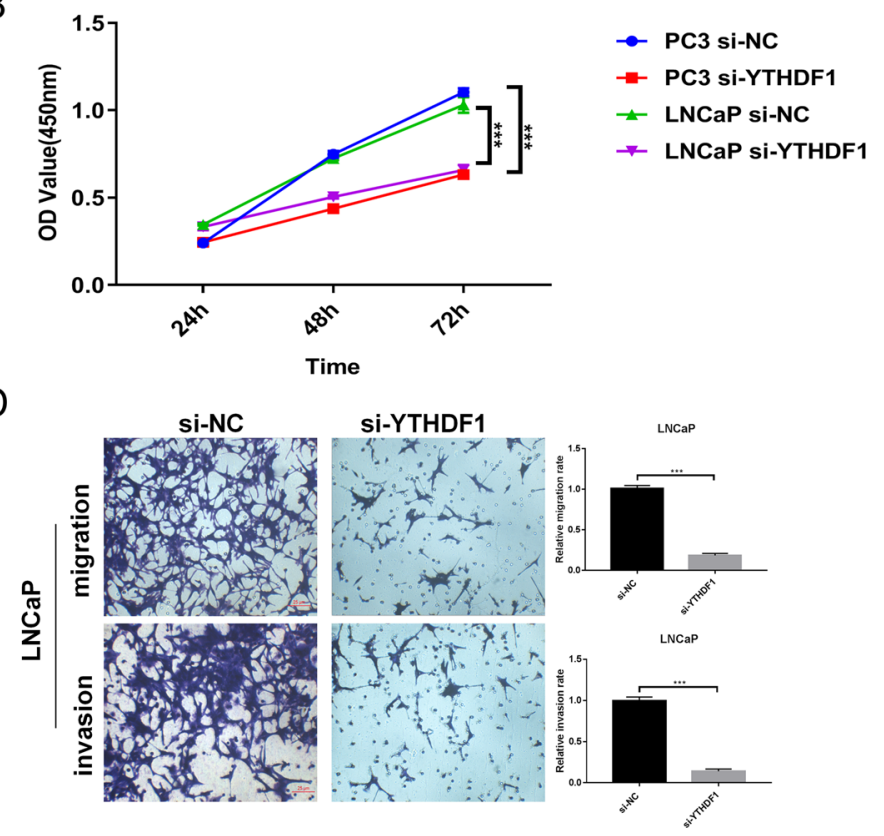

cell migration and invasion ability after transfection of si-YTHDF1 for $48 \mathrm{~h}$. D LNCaP cells migration and invasion ability at $48 \mathrm{~h}$ after si-YTHDF1 transfection. Scale bar $50 \mu \mathrm{m}$, the magnification is 200 times. $* \mathrm{P}<0.05$, $* * \mathrm{P}<0.005, * * * \mathrm{P}<0.001$

TRIM44 in PC3 cells, the level of TRIM44 was up-regulated, indicating a successful transfection. To further verify the expression of TRIM44, we performed WB experiments. The results indicated that after overexpressing TRIM44 in PC 3 cells, the expression level of TRIM44 protein was significantly up-regulated (Fig. 4B). Cell function experiments showed that cell proliferation, migration, and invasion ability were restored after overexpressing TRIM44 in PC3 cells (Fig. 4C, D). This suggests that YTHDF1 promotes PCa cells proliferation, migration, and invasion by regulating TRIM44.

\section{Discussion}

PCa is regarded as a common cancer with a high mortality rate in men (Jain and Sapra 2021). Current markers used for PCa detection, such as prostate-specific antigen screening, lead to significant overtreatment (Loeb and Giri 2021). As a reversible epigenetic modification, $\mathrm{m} 6 \mathrm{~A}$ is associated with the occurrence, metastasis, and drug resistance of tumors (Huang et al. 2020). YTHDF1 plays an essential role in a variety of cancers as a core factor of RNA methylation modification (Yarmishyn et al. 2020; Zhang et al. 2020). However, its specific mechanism in PCa has not been defined. In the present study, we analyzed YTHDF1 expression in PCa by querying the TCGA database and found that YTHDF1 

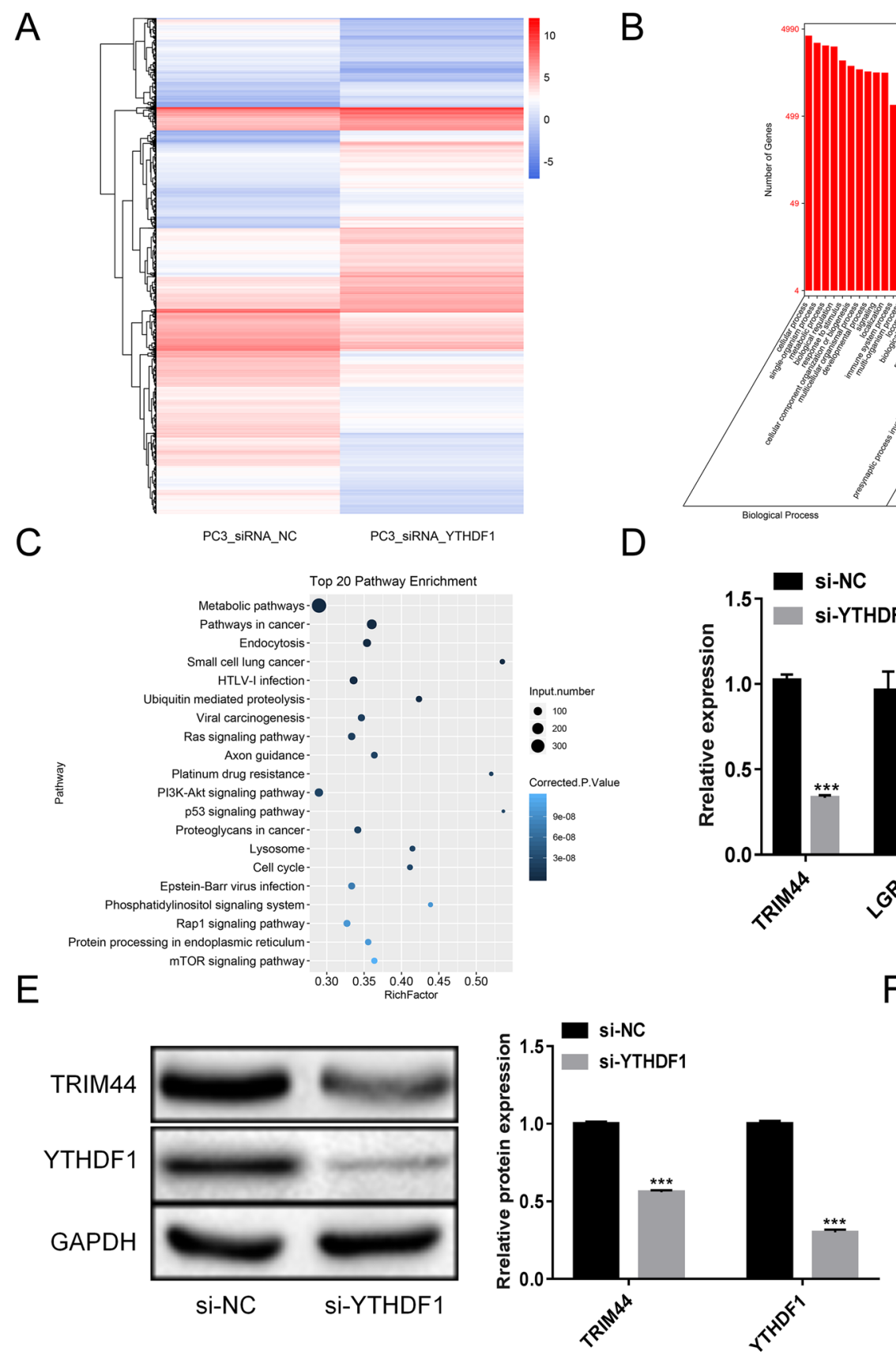

B

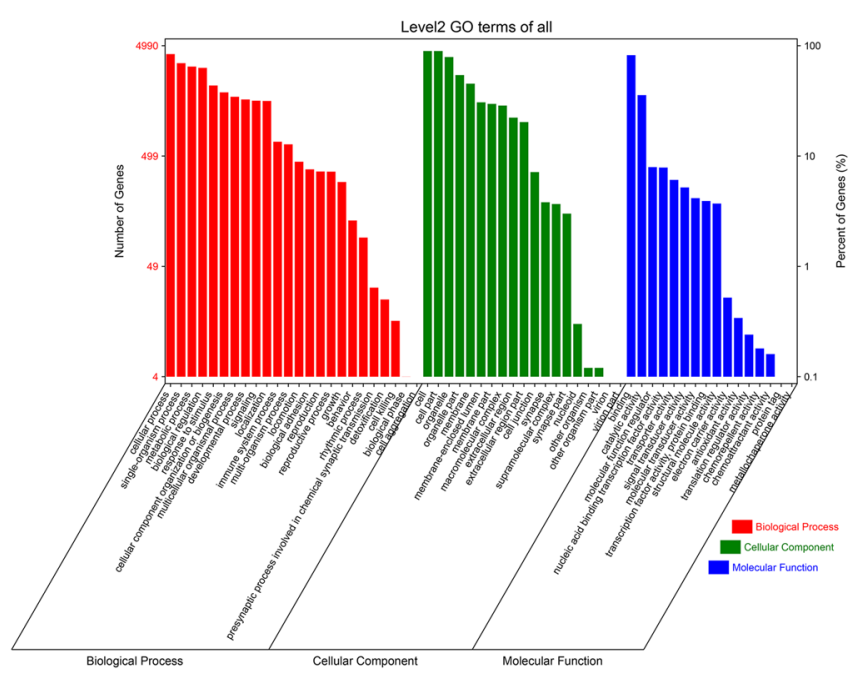

$\mathrm{D}$

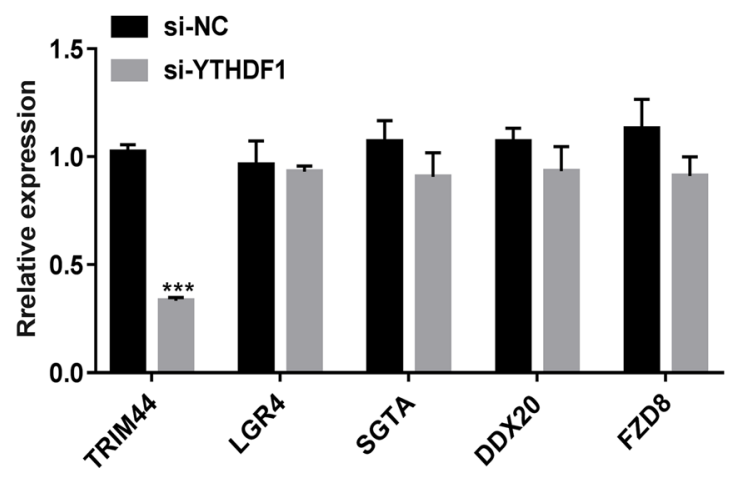

$\mathrm{F}$

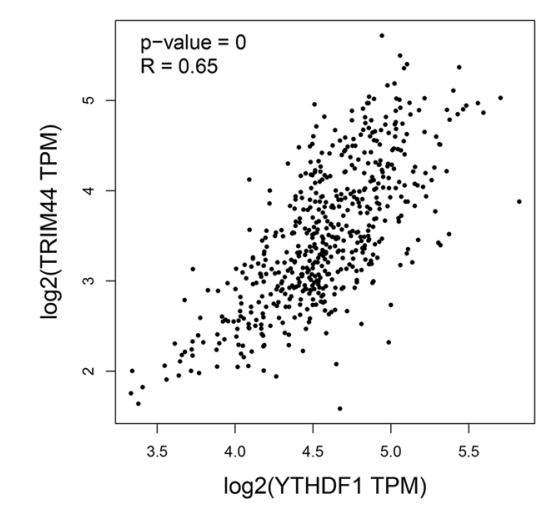

Fig. 3 YTHDF1 regulatory mechanism in prostate cancer as analyzed by mRNA sequencing. A Analysis of mRNA differential expression in PC3_siRNA_NC and PC3_siRNA_YTHDF1 groups. B GO analysis. C KEGG analysis. D The levels of TRIM44, LGR4, SGTA,

was highly expressed in PCa tissues. PCa patients with high expression of YTHDF1 had a relatively poor prognosis, indicating that it may function as a proto-oncogene.

YTHDF1 is a "reader" of m6A-modified mRNA (Wang et al. 2015). The change in m6A levels may be involved in $\mathrm{PCa}$ occurrence and development (Wu et al. 2021). Previous studies have reported that YTHDF1 promotes the
DDX20, and FZD8 were measured by qRT-PCR. E Western blotting detection of TRIM44 and YTHDF1 levels. F The correlation between YTHDF1 and TRIM44. Scale bar $50 \mu \mathrm{m}$, the magnification is 200 times. $* \mathrm{P}<0.05, * * \mathrm{P}<0.005, * * * \mathrm{P}<0.001$

occurrence and metastasis of gastric cancer in an m6Adependent manner by promoting the translation of USP 14 and it may represent a potential target for gastric cancer treatment (Chen et al. 2021c). YTHDF1 aggravates cervical cancer progression through m6A-mediated RANBP2 upregulation (Wang et al. 2021). Overexpression of YTHDF1 can reduce the sensitivity of colon cancer cells 

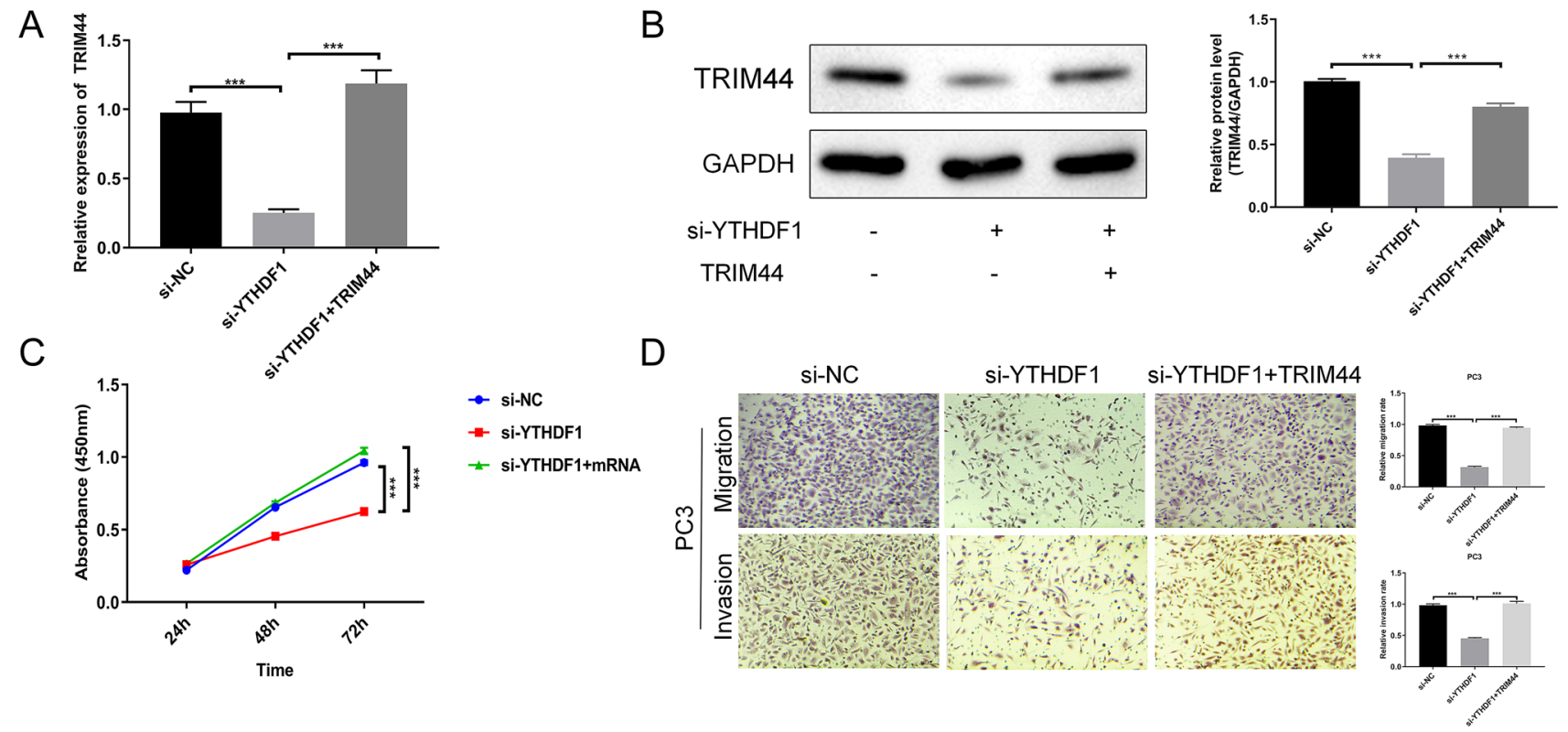

Fig. 4 YTHDF1 regulates TRIM44 to promote prostate cancer cells proliferation, migration, and invasion. A qRT-PCR determination of TRIM44 transfection efficiency. B Western blotting detection of TRIM44 protein levels. C CCK-8 assay was performed to detect PC3 cell proliferation at 24,48 , and $72 \mathrm{~h}$. D PC3 cell migration and invasion ability after transfection with TRIM 44 for $48 \mathrm{~h}$. Scale bar $50 \mu \mathrm{m}$, the magnification is 200 times. $* \mathrm{P}<0.05$, $* * \mathrm{P}<0.005$, *** $\mathrm{P}<0.001$ to cisplatin (Chen et al. 2021b). Consistent with previous studies, we found that YTHDF1 was highly expressed in prostate cancer cells and reduced expression of YTHDF1 inhibited $\mathrm{PCa}$ cell proliferation, migration, and invasion, suggesting that YTHDF1 may represent a target for PCa treatment.

The most common cause of death from PCa is metastasis (Krishna and Bergan 2014). TRIM44 plays an essential role in cancer progression. Overexpression of TRIM44 promotes cell proliferation and enhances hepatocellular carcinoma cell invasion and migration (Zhu et al. 2016). Knocking down TRIM44 inhibits the proliferation of renal cell carcinoma (Yamada et al. 2020). In PCa, TRIM44 gene knockout can inhibit PCa cell proliferation and invasion (Tan et al. 2017). Our results showed that YTHDF1 and TRIM44 were significantly correlated in PCa and they were both up-regulated. This suggests that YTHDF1 may promote PCa cell proliferation, migration, and invasion by modulating the expression of TRIM44. Therefore, we performed recovery experiments to confirm that YTHDF1 promotes $\mathrm{PCa}$ cell proliferation, migration, and invasion by regulating TRIM44. This study provides new insight into PCa etiology and progression. However, there are still limitations to our study. It is a basic in vitro study, which provides only a preliminary discussion on the molecular mechanism of YTHDF1 and TRIM44. Therefore, further studies in animals will be needed.

\section{Conclusions}

This study provided evidence that YTHDF1 promotes $\mathrm{PCa}$ cells proliferation, migration, and invasion by regulating TRIM44. Defining the molecular mechanism through which YTHDF1 regulates TRIM44 may be helpful to identify potential targets for PCa treatment to control its growth and metastasis.

Acknowledgements This project was funded by the Medical Research Foundation of Qingyuan People's Hospital under Grant numbers 20190205 and 20190206.

Author contributions JZ conceived and designed the study and critically revised the manuscript. WL and GC performed the experiments, analyzed the data, and drafted the manuscript. ZF, BZ, LZ, YZ, JM and CJ participated in the study design, study implementation, and manuscript revision. All authors read and approved the final manuscript.

Funding This work was supported by the grants from the Natural Science Foundation of Guangdong Province under Grant numbers 2016A030307033 to J.Z., and 2019A1515011107 to B.Z.; the National Natural Science Foundation of China under Grant numbers 81900688 to B.Z. and 81802551 to Y.Z.; Chinese Postdoctoral Science Foundation under Grant numbers 2020M672593 to Y.Z.; the Medical Research Foundation of Guangdong Province under Grant numbers A2019473 to B.Z. and B2020011 to Y.Z.; and the Science and Technology Foundation of Qingyuan City under Grant number 2020KJJH009 to B.Z. 


\section{Declarations}

Conflict of interest The authors declare no conflicts of interest in this study.

Open Access This article is licensed under a Creative Commons Attribution 4.0 International License, which permits use, sharing, adaptation, distribution and reproduction in any medium or format, as long as you give appropriate credit to the original author(s) and the source, provide a link to the Creative Commons licence, and indicate if changes were made. The images or other third party material in this article are included in the article's Creative Commons licence, unless indicated otherwise in a credit line to the material. If material is not included in the article's Creative Commons licence and your intended use is not permitted by statutory regulation or exceeds the permitted use, you will need to obtain permission directly from the copyright holder. To view a copy of this licence, visit http://creativecommons.org/licenses/by/4.0/.

\section{References}

Chen L, Yi C, Li W, Tseng Y, Zhang J, Liu J (2021a) Inhibition of SPATS2 suppresses proliferation and invasion of hepatocellular carcinoma cells through TRIM44-STAT3 signaling pathway. J Cancer 12:89-98

Chen P, Liu XQ, Lin X, Gao LY, Zhang S, Huang X (2021b) Targeting YTHDF1 effectively re-sensitizes cisplatin-resistant colon cancer cells by modulating GLS-mediated glutamine metabolism. Mol Ther Oncolytics 20:228-239

Chen XY, Liang R, Yi YC, Fan HN, Chen M, Zhang J, Zhu JS (2021c) The $\mathrm{m}(6) \mathrm{A}$ reader YTHDF1 facilitates the tumorigenesis and metastasis of gastric cancer via USP14 translation in an $\mathrm{m}(6)$ A-dependent manner. Front Cell Dev Biol 9:647702

Haberkorn U, Eder M, Kopka K, Babich JW, Eisenhut M (2016) New strategies in prostate cancer: prostate-specific membrane antigen (PSMA) ligands for diagnosis and therapy. Clin Cancer Res 22:9-15

Huang H, Weng H, Chen J (2020) M(6)A modification in coding and non-coding RNAs: roles and therapeutic implications in cancer. Cancer Cell 37:270-288

Jain MA, Sapra A (2021) Cancer prostate screening. In: StatPearls. StatPearls Publishing, Treasure Island

Jemal A, Siegel R, Ward E, Murray T, Xu J, Thun MJ (2007) Cancer statistics, 2007. CA Cancer J Clin 57:43-66

Krishna SN, Bergan RC (2014) Therapeutic modulation of prostate cancer metastasis. Future Med Chem 6:223-239

Lancia A, Zilli T, Achard V, Dirix P, Everaerts W, Gomez-Iturriaga A, Ingrosso G, Liefhooghe N, Miralbell R, Siva S et al (2019) Oligometastatic prostate cancer: the game is afoot. Cancer Treat Rev 73:84-90

Li J, Meng S, Xu M, Wang S, He L, Xu X, Wang X, Xie L (2018) Downregulation of $\mathrm{N}(6)$-methyladenosine binding YTHDF2 protein mediated by miR-493-3p suppresses prostate cancer by elevating N(6)-methyladenosine levels. Oncotarget 9:3752-3764

Liang Y, Zhan G, Chang KJ, Yang YP, Wang L, Lin J, Hsu CH (2020) The roles of m6A RNA modifiers in human cancer. J Chin Med Assoc 83:221-226

Liu S, Meng F, Ding J, Ji H, Lin M, Zhu J, Ma R (2019) High TRIM44 expression as a valuable biomarker for diagnosis and prognosis in cervical cancer. Biosci Rep 39:BSR20181639

Liu T, Wei Q, Jin J, Luo Q, Liu Y, Yang Y, Cheng C, Li L, Pi J, Si Y et al (2020) The m6A reader YTHDF1 promotes ovarian cancer progression via augmenting EIF3C translation. Nucleic Acids Res 48:3816-3831
Loeb S, Giri VN (2021) Clinical implications of germline testing in newly diagnosed prostate cancer. Eur Urol Oncol 4:1-9

Meyer KD, Jaffrey SR (2017) Rethinking m(6)A readers, writers, and erasers. Annu Rev Cell Dev Biol 33:319-342

Nguyen-Nielsen M, Borre M (2016) Diagnostic and therapeutic strategies for prostate cancer. Semin Nucl Med 46:484-490

Nishizawa Y, Konno M, Asai A, Koseki J, Kawamoto K, Miyoshi N, Takahashi H, Nishida N, Haraguchi N, Sakai D et al (2018) Oncogene c-Myc promotes epitranscriptome m(6)A reader YTHDF1 expression in colorectal cancer. Oncotarget 9:7476-7486

Sato J, Azuma K, Kinowaki K, Ikeda K, Ogura T, Takazawa Y, Kawabata H, Kitagawa M, Inoue S (2021) Combined A20 and tripartite motif-containing 44 as poor prognostic factors for breast cancer patients of the Japanese population. Pathol Int 71:60-69

Shi Y, Fan S, Wu M, Zuo Z, Li X, Jiang L, Shen Q, Xu P, Zeng L, Zhou $Y$ et al (2019) YTHDF1 links hypoxia adaptation and non-small cell lung cancer progression. Nat Commun 10:4892

Sun T, Wu R, Ming L (2019) The role of m6A RNA methylation in cancer. Biomed Pharmacother 112:108613

Tan Y, Yao H, Hu J, Liu L (2017) Knockdown of TRIM44 inhibits the proliferation and invasion in prostate cancer cells. Oncol Res 25:1253-1259

Tian C, Deng Y, Jin Y, Shi S, Bi H (2018) Long non-coding RNA RNCR3 promotes prostate cancer progression through targeting miR-185-5p. Am J Transl Res 10:1562-1570

Wang X, Zhao BS, Roundtree IA, Lu Z, Han D, Ma H, Weng X, Chen K, Shi H, He C (2015) N(6)-Methyladenosine modulates messenger RNA translation efficiency. Cell 161:1388-1399

Wang H, Luo Q, Kang J, Wei Q, Yang Y, Yang D, Liu X, Liu T, Yi P (2021) YTHDF1 aggravates the progression of cervical cancer through m(6)A-mediated up-regulation of RANBP2. Front Oncol 11:650383

Wu Q, Xie X, Huang Y, Meng S, Li Y, Wang H, Hu Y (2021) N6-Methyladenosine RNA methylation regulators contribute to the progression of prostate cancer. J Cancer 12:682-692

Xia TL, Li X, Wang X, Zhu YJ, Zhang H, Cheng W, Chen ML, Ye Y, Li Y, Zhang A et al (2021) N(6)-Methyladenosine-binding protein YTHDF1 suppresses EBV replication and promotes EBV RNA decay. EMBO Rep 22:e50128

Yamada Y, Kimura N, Takayama KI, Sato Y, Suzuki T, Azuma K, Fujimura T, Ikeda K, Kume H, Inoue S (2020) TRIM44 promotes cell proliferation and migration by inhibiting FRK in renal cell carcinoma. Cancer Sci 111:881-890

Yarmishyn AA, Yang YP, Lu KH, Chen YC, Chien Y, Chou SJ, Tsai PH, Ma HI, Chien CS, Chen MT et al (2020) Musashi-1 promotes cancer stem cell properties of glioblastoma cells via upregulation of YTHDF1. Cancer Cell Int 20:597

Zhang Y, Liu X, Liu L, Li J, Hu Q, Sun R (2020) Expression and prognostic significance of m6A-related genes in lung adenocarcinoma. Med Sci Monit 26:e919644

Zhang Z, Wang Q, Zhang M, Zhang W, Zhao L, Yang C, Wang B, Jiang K, Ye Y, Shen Z et al (2021) Comprehensive analysis of the transcriptome-wide m6A methylome in colorectal cancer by MeRIP sequencing. Epigenetics 16:425-435

Zhao X, Chen Y, Mao Q, Jiang X, Jiang W, Chen J, Xu W, Zhong L, Sun X (2018) Overexpression of YTHDF1 is associated with poor prognosis in patients with hepatocellular carcinoma. Cancer Biomark 21:859-868

Zhao W, Cui Y, Liu L, Ma X, Qi X, Wang Y, Liu Z, Ma S, Liu J, Wu J (2020) METTL3 facilitates oral squamous cell carcinoma tumorigenesis by enhancing c-Myc stability via YTHDF1-mediated $\mathrm{m}(6)$ A modification. Mol Ther Nucleic Acids 20:1-12

Zhu X, Wu Y, Miao X, Li C, Yin H, Yang S, Lu X, Liu Y, Chen $Y$, Shen R et al (2016) High expression of TRIM44 is associated with enhanced cell proliferation, migration, invasion, and 
resistance to doxorubicin in hepatocellular carcinoma. Tumour Biol 37:14615-14628
Publisher's Note Springer Nature remains neutral with regard to jurisdictional claims in published maps and institutional affiliations. 